\title{
Zur Logik der Weltbildanalyse in Georg Simmels Philosophie des Geldes
}

\author{
Zur Logik der Weltbildanalyse in Georg Simmels "Philosophie des Geldes" \\ von Klaus Lichtblau
}

I.

Georg Simmels Werk hat in den vergangenen zehn Jahren eine bemerkenswerte internationale Renaissance erfahren. Diese auch in den Bemühungen um eine Gesamtausgabe zum Ausdruck kommende Aktualität seines Denkens steht dabei in einem engen Zusammenhang mit der wirkungsgeschichtlichen Bedeutung, die seinen Arbeiten schon zu Lebzeiten von vielen seiner Zeitgenossen zuerkannt worden ist. Insbesondere die Entwicklung der deutschsprachigen Soziologie seit der Jahrhundertwende ist nur schwer ohne eine Berücksichtigung der wegweisenden Funktion seiner "Philosophie des Geldes" und seiner verschiedenen kulturphilosophischen Essays in ihrer Eigenart zu verstehen. Insofern ist dem Urteil von Georg Lukács aus dem Jahre 1919 voll zuzustimmen, wenn er schreibt: "Eine Soziologie der Kultur, wie sie von Max Weber, Troeltsch, Sombart und anderen unternommen wird, ist - so sehr sie alle auch methodisch von ihm abweichen mögen - doch nur auf dem von ihm geschaffenen Boden möglich geworden." Walter Benjamin hat darüber hinaus in Georg Simmel zurecht einen "Ahnen des Kulturbolschewismus" gesehen, dessen kulturkritische Schriften viele zentrale Motive des "westlichen Marxismus" und der "Kritischen Theorie" vorweggenommen haben. Augenscheinlich ist dabei in beiden Rezeptionsprozessen jedoch eine zentrale Unterscheidung zwischen "Inhalt" und "Form" von Simmels Analyse der modernen Geldwirtschaft und ihrer verschiedenen kulturellen Erscheinungsformen vorgenommen worden, die mit starken methodologischen bzw. "ideologiekritischen" Bedenken gegenüber seiner eigenen Untersuchungsmethode verbunden gewesen ist. Insofern muß nach wie vor davon ausgegangen werden, daß insbesondere der enorme wirkungsgeschichtliche Einfluß von Simmels "Philosophie des Geldes" uns nur wenig direkten Aufschluß über deren spezifischen kognitiven Status zu geben vermag.

Dieser Sachverhalt soll im folgenden nur exemplarisch an einem Beispiel veranschaulicht werden: Während Werner Sombart und Max Weber an einer Untersuchung der Bedeutung des "kapitalistischen Geistes" für die Genese der modernen Erwerbswirtschaft in Gestalt einer historischen Kausalanalyse interessiert gewesen sind, hat Simmel seiner "Philosophie des Geldes" ein solches historiographisches Interesse ausdrücklich abgesprochen; denn er hat seine Aufmerksamkeit primär auf eine Analyse der inneren "Bedeutsamkeit" der modernen Geldwirtschaft und der in ihr zum Ausdruck kommenden "Wertgefühle" gerichtet. Dem Umstand, daß Simmel damit zugleich grundsätzlich eine ahistorische Form der Analyse wählte, muß insofern ein größeres Gewicht zugesprochen werden als z.B. dem von Max Weber geäußerten Vorwurf, daß Simmel nicht zureichend zwischen Geldwirtschaft im allgemeinen und dem modernen Kapitalismus im Sinne einer rational strukturierten und betriebsförmig geordneten Arbeitsorganisation unterschieden habe.

Diese Differenz zwischen dem Projekt einer historisch-kulturwissenschaftlichen Analyse der Genese des modernen Kapitalismus, wie es unter anderem von Sombart und Max Weber verfolgt worden ist, einerseits und dem spezifischen kognitiven Status von Simmels "Philosophie des Geldes" andererseits beinhaltet zugleich eine unterschiedliche Einschätzung des Verhältnisses zwischen einer einzelwissenschaftlich verfahrenden Wirklichkeits-erkenntnis und einem umfassenden philosophischen Deutungsanspruch, die uns etwas mehr Klarheit über Simmels eigentliche Absicht zu geben vermag. Während Max Webers Werk nämlich durch eine grundsätzliche Skepsis gegenüber der Möglichkeit eines einheitlichen Verständnisses der modernen Welt geprägt war, wie es einst in den verschiedenen religiösen und metaphysischen Weltbildern zum Ausdruck kam, ist Simmel gerade an einer Klärung der Bedingungen für die Entwicklung eines den spezifischen Erfahrungsgehalten der Moderne gerecht werdenden philosophischen Weltbildes interessiert. Webers Plädoyer für ein "nachmetaphysisches Denken" steht insofern durchaus in einem spannungsreichen Verhältnis zu Simmels 
Gleichwohl war auch Simmel der Ansicht, daß sich eine solch spezifisch "moderne" Metaphysik nicht mehr ohne weiteres axiomatisch durchführen läßt, sondern vor dem Hintergrund des fortgeschrittenen Erkenntnisstandes der Einzelwissenschaften zu bewähren habe. Simmel hat in diesem Zusammenhang sowohl eine "untere" als auch eine "obere" Grenze jeder einzelwissenschaftlichen Wirklichkeitserkenntnis angegeben, um deutlich zu machen, welche Erkenntnisleistungen grundsätzlich nicht von den Einzelwissenschaften bewältigt werden können, sondern nach wie vor den legitimen Gegenstand einer genuin philosophischen Reflexion bilden. Denn zum einen ist jede einzelwissenschaftliche Erkenntnis auf begriffliche und methodische Voraussetzungen angewiesen, deren apriorischen Charakter sie selbst nicht weiter begründen kann und die deshalb im Rahmen einer Erkenntnistheorie der jeweiligen Einzelwissenschaft analysiert und beschrieben werden müssen. Zum anderen ist ihm zufolge eine einzelwissenschaftlich verfahrende Form der Erkenntnis aufgrund ihres fragmentarischen Charakters aus prinzipiellen Gründen nicht in der Lage, die einzelnen Inhalte des positiven Wissens "durch abschließende Begriffe zu einem Weltbild zu ergänzen und auf die Ganzheit des Lebens zu beziehen". Diese Aufgabe bildet für ihn deshalb den Gegenstand einer "philosophischen Spekulation" bzw. einer Metaphysik der entsprechenden Einzelwissenschaft, deren bleibender formaler Wert sich gerade dadurch auszeichne, "überhaupt ein vollendetes Weltbild nach durchgehenden Prinzipien anzustreben". Wissenschaft und Philosophie sind Simmel zufolge insofern komplementär aufeinander bezogen: Als "Anticipation des realistischen Erkennens" bildet die philosophische Spekulation nämlich zum einen einen heuristischen Vorgriff auf ein mögliches positives Wissen, das durch den Fortschritt der einzelwissenschaftlichen Wirklichkeitserkenntnis prinzipiell auch einer "exakten" Form des Denkens zugänglich gemacht werden kann; andererseits ermögliche die Philosophie zugleich auf symbolischem Wege die Befriedigung eines metaphysischen Bedürfnisses, die uns in einer "realistischen" Form versagt bleibe.

Nicht zufällig betont Simmel dabei die enge "Wahlverwandtschaft" zwischen dem spezifischen Bedeutungsgehalt eines großen Kunstwerks und der Eigenart einer modernen metaphysischen Weltbetrachtung. Denn während die traditionellen philosophischen Systeme unmittelbar auf eine Interpretation der "Gesamtheit des Daseins" ausgerichtet waren, geht seine "Philosophie des Geldes" ähnlich wie die Kunst von einem einzelnen Gegenstand aus, um diesem dann schließlich "durch seine Erweiterung und Hinausführung zur Totalität und zum Allgemeinsten gerecht zu werden". Insofern kann Simmel auch zurecht sagen, daß keine Zeile seiner groß angelegten Untersuchung über das Wesen der modernen Geldwirtschaft in einem engeren nationalökonomischen Sinne zu verstehen sei; denn zum einen beabsichtige sie eine Darstellung der wirtschaftlichen Formen als "Ergebnis tieferer Wertungen und Strömungen, psychologischer, ja metaphysischer Voraussetzungen" und zum anderen eine Einbeziehung des wirtschaftlichen Lebens in die "Ursachen der geistigen Kultur". Gleichwohl bleibt zu klären, warum Simmel das Geld zum Ausgangspunkt für die Entwicklung eines "prinzipiell bestimmten Weltbildes" nahm, um damit zugleich die allgemeine Signatur des modernen Zeitalters zu verdeutlichen. Und es ist zu fragen, welche spezifische "Logik" bzw. methodischen Prämissen mit einer solchen Weltbildanalyse verbunden sind, die zugleich beansprucht, das Erbe der metaphysischen Tradition für eine philosophische Deutung der Moderne fruchtbar zu machen.

II.

Unter "Weltbild" versteht Simmel eine einheitliche Interpretation des Seins, die umfassend genug ist, um der "Ganzheit des Lebens" ausgehend von jeweils einem der großen Gegensatzpaare, welche die Geschichte des menschlichen Denkens geprägt haben, in einer spezifischen Weise gerecht zu werden. Die Erfahrung von Gegensätzen und Konflikten, die das Leben als solches kennzeichnen, ist dabei sowohl reale als auch logische Voraussetzung für einen grundsätzlichen Pluralismus der Weltbilder, über den nicht doktrinär entschieden werden kann, da in ihm spezifische Wertempfindungen zum Ausdruck kommen, die zugleich prinzipiell mögliche Einstellungen zur Welt kennzeichnen. Gegenüber der Einseitigkeit einer rein materialistischen Weltanschauung ist eine idealistische somit im gleichen Recht. Entscheidend bleibt, daß ein solches Weltbild dem ihm jeweils zugrundeliegenden zentralen Gegensatz überhaupt in einer logisch befriedigenden Weise Rechnung zu tragen 
vermag. So zeichnen sich zum Beispiel die in großen Übergangsepochen geprägten Weltbilder dadurch aus, daß sie den historischen Gegensatz zwischen dem "Alten" und dem "Neuen" in einer für ihre Zeit charakteristischen Form verarbeitet haben. Simmel nennt darüber hinaus aber auch den Gegensatz zwischen dem Materiellen und dem Ideellen, dem Verstandesmäßigen und dem Willensmäßigen, dem Absoluten und dem Relativen sowie andere Gegensatzpaare als mögliche Pole, die weit genug gespannt sind, "um ein Weltbild darein zu fassen".

Ausgangspunkt von Simmels "Philosophie des Geldes" bildet die Unterscheidung zwischen zwei fundamental verschiedenen Formen der Weltorientierung, die auf den Dualismus zwischen einer theoretischen Einstellung zur Welt und dem praktischen Sichverhalten in der Welt zurückzuführen sind. Das durch die Vorherrschaft des Intellekts geprägte naturwissenschaftliche Weltbild beruht demzufolge auf einer Auffassung der Wirklichkeit, in der die Inhalte dieser Welt unterschiedslos der Herrschaft des Naturgesetzes unterworfen sind. Diese Gleichgültigkeit und Indifferenz der natürlichen Welt wird Simmel zufolge erst durchbrochen, wenn wir sie nicht mehr unter dem Gesichtspunkt ihrer objektiven Wirklichkeit, sondern im Hinblick auf ihren möglichen Wert bezüglich unserer Bedürfnisse und Interessen betrachten. Indem wir bestimmten Dingen einen Wert zusprechen, betonen wir nämlich gerade den Unterschied, der sie im Rahmen unserer Wertpräferenzen gegenüber anderen Gegenständen auszeichnet. Jede Bewertung von Gegenständen ist insofern an die Existenz einer Rangordnung der Werte gebunden, die zugleich einen Aufschluß über unser subjektives Wertempfinden gibt. Der psychologische Vorgang des Wertens bildet dabei einerseits selbst noch einen Bestandteil der natürlichen Welt; die mit dieser Bewertung verbundene inhaltliche Bedeutung ist dagegen "etwas dieser Welt unabhängig Gegenüberstehendes, und so wenig ein Stück ihrer, daß es vielmehr die ganze Welt ist, von einem besonderen Gesichtspunkt angesehen".

Diese prinzipielle Möglichkeit der Wertung stellt Simmel zufolge ein nicht weiter begründbares Vermögen bzw. "Urphänomen" dar. Aufgrund ihres fundamentalen Charakters kommt der Kategorie des Wertes ähnlich wie der Kategorie des Seins insofern eine grundlegende Funktion im Rahmen der Konstitution unseres Weltbildes zu. Simmel unterscheidet deshalb zwischen der intellektuellen Struktur unseres Weltbildes, in dem sich die Entwicklung des theoretischen Denkens widerspiegelt, und dem durch unser Wertempfinden geprägten Gehalt dieses Weltbildes, der sich im wesentlichen unserer willensmäßigen Praxis innerhalb der natürlich vorgegebenen Welt verdankt. Dies heißt nicht, daß diese beiden Formen der Ausgestaltung unseres Weltbildes beziehungslos zueinander stehen müßten, wohl aber, daß der Entwicklung der Kategorie des wirtschaftlichen Wertes im Rahmen einer "Philosophie des Geldes" die Funktion einer "Weltformel" zugesprochen bekommt, in der sich die Einheit unserer theoretischen Erkenntnis und willensmäßigen Praxis am umfassendsten widerspiegelt. Ich möchte im folgenden deshalb zunächst auf die Parallelität in Simmels Darstellung der intellektuellen und der praktischen Dimension des von ihm rekonstruierten modernen Weltbildes eingehen, bevor ich anschließend die übergreifende Funktion und den symbolischen Gehalt des Geldes für ein adäquates Verständnis der Moderne zu charakterisieren versuche.

III.

Simmel sieht eine grundlegende "Analogie" bzw. "Korrelation" zwischen dem Rationalismus und Intellektualismus des durch die modernen Naturwissenschaften geprägten Weltbildes und der spezifischen Form der Objektivität des wirtschaftlichen Wertes gegeben, wie sie innerhalb der entfalteten Geldwirtschaft zum Ausdruck kommt. Sowohl die wissenschaftliche Form der Naturerkenntnis als auch die ökonomische Praxis des Warentausches sind durch eine "evolutionistische Beziehung zwischen Subjekt und Objekt" geprägt, in welcher sich die vormals substantielle Einheit des antiken Weltbildes in die selbständigen Extreme des Subjektiven und des Objektiven ausdifferenziert hat. Innerhalb der teleologischen Struktur des menschlichen Handelns ist die gefühlsmäßige und affektuelle Dimension des Willensprozesses notwendig an die Setzung eines Endzweckes gebunden, während die Entwicklung der verstandesmäßigen Funktionen mit dem zunehmenden Einsatz der Mittel einhergeht, die zur Erreichung des gewünschten Endzweckes erforderlich sind. Durch den Gebrauch des Geldes als allgemeinem Medium des ökonomischen Austausches verlängern sich die einzelnen Handlungsverkettungen schließlich zu einem "ungeheuren teleologischen Zusammenhang", dessen logische Struktur sich dabei der des 
"naturgesetzlichen Kosmos" nähert. Denn indem das Geld alle Gegenstände des praktischen Bedarfs auf ihren rein ökonomischen Wert reduziert, erscheint es wie der naturwissenschaftliche Begriff der Energie als ein inhaltlich völlig abstrakter Vergleichsmaßstab, dessen "Charakterlosigkeit" gerade darin besteht, die getauschten Gegenstände allein nach Maßgabe ihrer quantitativen Bestimmtheit zur Geltung kommen zu lassen.

Diese Gleichgültigkeit gegenüber der individuellen Eigenheit teilt aber das Geld mit den logischen Funktionen des menschlichen Intellekts, deren "Form arithmetischer Genauigkeit" auf die Welt der Dinge selbst "zurückstrahlen" muß, wenn sie als Maxime des praktischen Handelns zur Anwendung gelangt. Simmel läßt es dabei bewußt offen, ob sich der spezifisch rationalistische Charakter der Neuzeit ausschließlich der formalen Bestimmtheit des Geldes verdankt oder ob in diesem Zusammenhang nicht auch von einer eigenständigen Entwicklung des naturwissenschaftlichen Weltbildes ausgegangen werden muß, wenn er schreibt: "Erst die Geldwirtschaft hat in das praktische Leben - und wer weiß, ob nicht auch in das theoretische - das Ideal zahlenmäßiger Berechenbarkeit gebracht." Gegenüber einer eindeutigen kausalgenetischen Hypothese zieht es Simmel denn auch vor, eher von einer formalen "Analogie" bzw. einer "Korrelation" oder "Wechselwirkung" zwischen dem Geld und dem Intellekt zu sprechen, die zugleich auch einen "Hinweis auf ein tiefer gelegenes, ihnen gemeinsames Prinzip" zu geben vermag, "das die Gleichheit ihrer Entwicklung trägt".

Diesen Parallelismus zwischen der Entwicklung der Geldwirtschaft und der theoretisch-naturwissenschaftlichen Form des Denkens versucht Simmel anhand der Genese einer beide Sphären umfassenden Weltbildstruktur aufzuzeigen, die zugleich die spezifische Eigenart der okzidentalen Moderne gegenüber anderen Epochen und Kulturen deutlich machen soll. Simmel argumentiert auch in diesem Zusammenhang nicht historisch-narrativ, sondern führt einen Strukturvergleich durch, um das traditionelle, antik-mittelalterliche Weltbild von dem der Moderne begrifflich abzugrenzen und zu untersuchen.

Die antiken und mittelalterlichen Wert- und Preistheorien beruhten noch auf der Vorstellung, daß der ökonomische Wert einer Ware dieser gewissermaßen als etwas Objektives und Substantielles zugrundeläge, das auch unabhängig von einem konkreten Tauschverhältnis zu bestimmen sei. Jedem Tauschvorgang sollte - so lautete die entsprechende ethische Maxime - deshalb immer auch jener "gerechte Preis" zugrundegelegt werden, welcher mit dieser vorgängigen Bestimmtheit des Wertes einer Ware "an sich" übereinstimmt. Diese Wertauffassung entspricht in historischer Hinsicht einer Epoche, deren ökonomische Verhältnisse im wesentlichen durch die Naturalwirtschaft bestimmt sind bzw. die in dem Erwerb von Gebrauchsgütern, nicht aber in dem des Geldes als einem Selbstzweck den eigentlichen Sinn und das eigentliche Ziel des ökonomischen Tausches sieht. Sie ist zugleich Ausdruck eines substantiell-absolutistischen Weltbildes, das hinter allen Prozessen und Erscheinungsformen das Wirken einer "Kraft" sieht, die auch unabhängig von den zufälligen Beziehungen zwischen den Dingen bestimmbar ist und absolute Geltung für sich beanspruchen kann. Das Erkenntnisinteresse der traditionellen Metaphysik besteht deshalb darin, dieses "Absolute" aus der Mannigfaltigkeit der Phänomene und der zwischen ihnen stattfindenden Prozessen herauszuabstrahieren und letztere als die bloßen Erscheinungsformen desselben begreifbar zu machen.

Demgegenüber besteht die Eigenart des modernen Weltbildes gerade darin, diese "Festigkeit und Absolutheit der Weltinhalte" in reine Bewegungen und Relationen aufzulösen und der Vorstellung einer "absoluten Wahrheit" die eines "Stromes der ewigen Entwicklung" des menschlichen Denkens gegenüberzustellen. Simmel zeigt dabei exemplarisch auf, wie die moderne Erkenntnis- und Wissenschaftstheorie versucht, Kriterien für eine Objektivität des Denkens nicht mehr duch einen Rekurs auf vorgängige Wesensbestimmungen anzugeben, sondern rein intern als Relationen zwischen den einzelnen, sich wechselseitig bestimmenden Denkoperationen zu begründen: "Das Erkennen ist so ein freischwebender Prozeß, dessen Elemente sich gegenseitig ihre Stellung bestimmen. ... Daß unser Bild der Welt auf diese Weise >in der Luft schwebt<, ist nur in Ordnung, da ja unsere Welt selbst es tut."

Jener der Moderne eigentümliche, fundamentale Charakter der Relativität ist es denn auch, der Simmel dazu bewogen hat, eine Parallele zwischen dieser "Weltformel" und der "Formel des wirtschaftlichen Wertes" herzustellen. Denn auch das Geld ist dadurch gekennzeichnet, daß in ihm der Wert der Dinge, als ihre ökonomische "Wechselwirkung" verstanden, seinen "reinsten Ausdruck und Gipfel" gefunden hat: es ist der 
symbolische Ausdruck dessen, daß diese sich ihren Wert gegenseitig bestimmen und nur innerhalb dieses Verhältnisses als ökonomische Tatbestände gefaßt werden können. Damit fügt es sich aber zugleich bruchlos in das relativistische Weltbild der Moderne ein, dessen epochale und metaphysische Eigenart es seinerseits deutend zu erschließen hilft: "Dies ist die philosophische Bedeutung des Geldes: daß es innerhalb der praktischen Welt die entschiedenste Sichtbarkeit, die deutlichste Wirklichkeit der Formel des allgemeinen Seins ist, nach der die Dinge ihren Sinn aneinander finden ... Die reinste Wechselwirkung hat in ihm die reinste Darstellung gefunden, es ist die Greifbarkeit des Abstraktesten, das Einzelgebilde, das am meisten seinen Sinn in der Übereinzelheit hat."

Diese fundamentale Formentsprechung zwischen dem theoretischen Weltbild der Moderne und den praktischen Verhältnissen innerhalb der modernen Geldwirtschaft ist die tiefere Voraussetzung dafür, daß eine "Philosophie des Geldes" überhaupt den Anspruch auf eine umfassende epochale Deutung der Kultur der Moderne stellen kann. Denn sie verweist auf grundlegende Übereinstimmungen zwischen den logischen Formen unseres Intellekts und den Objektivationen unseres praktischen Handelns, die in Gestalt des Geldes einen adäquaten symbolischen Ausdruck gefunden haben. Als Verkörperung des Prinzips der Indifferenz, dessen funktionaler Wert allein in seiner permanenten Zirkulation begründet liegt, verkörpert das Geld mithin den Charakter des Rationalen und Logischen selbst, welcher die formale Einheitlichkeit und Geschlossenheit des modernen Weltbildes verbürgt. Um klären zu können, warum seine eigene Zirkulation zugleich zum Symbol der neuzeitlichen Erfahrung von Geschichte als einer "zeitlosen Bewegung" werden kann, soll im folgenden zunächst kurz Simmels Auffassung bezüglich der symbolischen Natur aller menschlichen Erkenntnis dargestellt werden, um daran anschließend seine Beschreibung des spezifischen temporalen Erfahrungsgehalts der Moderne innerhalb seiner Analyse des modernen Weltbildes zu charakterisieren.

IV.

Ausgangspunkt von Simmels Erörterung der spezifischen Erkenntnisleistung von Symbolisierungsprozessen bildet die Feststellung, daß die noch innerhalb des vormodernen Weltbildes verankerte metaphysische Einheit von Subjekt und Objekt im Rahmen der durch die moderne Geldwirtschaft geprägten Form der Vergesellschaftung durch eine evolutionistische Beziehung zwischen dem Bereich des Subjektiven und Objektiven ersetzt worden ist. Dieser Differenzierungsprozeß prägt dabei sowohl unsere theoretischen Formen der Erkenntnis als auch unser praktisches Verhalten in der Welt. Simmel zufolge kann man nämlich die verschiedenen Kulturstufen dahingehend charakterisieren und voneinander abgrenzen, in welchem Ausmaß sie sich der Vermittlung von Werkzeugen und Symbolen im Rahmen der zweckrationalen Orientierung des Handelns bedienen und gewissermaßen als Zwischenstufen in das Verhältnis des Menschen zu den ihn umgebenden Gegenständen einschieben. Im Unterschied zum reinen Werkzeug ist aber jeder Symbolisierung eigentümlich, daß sie zum einen eine abstrakte, d.h. intellektuelle Verknüpfung zwischen ihrer Ausdrucksgestalt und den durch sie dargestellten Inhalten bewirkt und daß sie zum anderen zugleich auf einer gegenseitigen symbolischen Deutung zwischen der gegenständlichen Erscheinungswelt und dem Bereich des Intelligiblen beruht. Simmel geht nämlich davon aus, daß uns sowohl unsere eigenen als auch fremdpsychische Erlebnisse nicht unmittelbar zugänglich sind, sondern allein vermittels räumlicher und zeitlicher "Symbole" bzw. "Analogien" beschrieben werden können. Andererseits würde auch die gegenständliche bzw. "äußere" Welt in eine Vielfalt von zusammenhangslosen Fragmenten zerfallen, würden wir sie nicht nach Maßgabe jenes "inneren Bildes" ordnen, das allein als Produkt spezifischer "seelischer" Funktionen zustandekommt. Insofern kann Simmel auch sagen, "daß die Seele das Bild der Gesellschaft und die Gesellschaft das Bild der Seele ist".

Simmels Rehabilitierung des Symbolischen steht dabei in der Tradition des von Goethe, Schleiermacher und den Romantikern geprägten Symbolverständnisses, demzufolge die "Idee" (das Allgemeine) immer nur in Gestalt eines Individuellen sinnlich in Erscheinung treten und dergestalt "symbolisch" erfaßt werden kann.

Bezeichnenderweise hatte auch Goethe diese entscheidende Neuprägung des Symbolbegriffs, der sich historisch aus der altprotestantischen Hermeneutik und Sakramentenlehre herleitet, zur Überwindung der "millionenfachen Hydra der Empirie" eingeführt und all jene Gegenstände als symbolisch bezeichnet, die in der Lage sind, uns in eine "sentimentalische Stimmung" zu versetzen: "Symbolische Gegenstände ... sind eminente Fälle, die in einer 
charakteristischen Mannigfaltigkeit als Repräsentanten von vielen anderen darstehen, eine gewisse Totalität in sich schließen und so von außen als von innen an eine gewisse Einheit und Allheit Anspruch machen".

Goethes Symbolverständnis muß dabei vor dem Hintergrund seines pantheistischen Weltbildes gesehen werden, das von Simmel in Gestalt eines ästhetischen Pantheismus in modifizierter Form übernommen worden ist. Simmel schließt dabei zugleich an die Ästhetik Johannes Volkelts an, derzufolge in der "Wechselwirkung" von Geist und Natur bzw. "Innenwelt" und "Außenwelt" sowie in der dadurch gegebenen Möglichkeit einer symbolischen Interpretation der gesamten gegenständlichen Erscheinungswelt der spezifische Geltungsanspruch eines "philosophischen Pantheismus" begründet liegt. Ist so im Rahmen einer solchen pantheistischen Weltanschauung die Möglichkeit gegeben, bezüglich jeder einzelnen empirischen Erscheinungsform zugleich den ihr zugrunde-liegenden "Typus" aufzuzeigen und allen Gegenständen des Alltags bzw. Manifestationen des modernen Lebens zugleich eine ästhetische Bedeutsamkeit zuzusprechen, so besteht die Eigenart des ihr entgegengesetzten "ästhe-tischen Individualismus" gerade darin, diese "Indifferenz" durch eine Betonung der "Rangordnung der Werte" zu ersetzen, welche gerade durch den spezifischen "Abstand" zwischen dem jeweiligen "Wert der Dinge" definiert ist. Simmel sieht diesen Gegensatz zwischen dem ästhetischen Individualismus und dem ästhetischen Pantheismus gerade als Kennzeichen der modernen Kultur an, welches zum einen die gesellschaftliche Bedeutung des "großen Kunstwerks" innerhalb einer autonomen ästhetischen Wertsphäre und zum anderen die ästhetische Bedeutung der verschiedenen kulturellen Manifestationen des modernen Lebens begründet. Beide ästhetische Ausdrucksformen sind ihm zufolge gleichermaßen einer "symbolischen Deutung" zugänglich. Denn "Symbolik" ist ihm zufolge das eigentliche "Grundwesen aller Kunst".

Simmel hat diese Auffassung bezüglich des symbolischen Charakters aller kulturellen Objektivationen in seiner "Philosophie des Geldes" am systematischsten begründet. Es gelingt ihm dabei, an einem "einzelnen Beispiel" zugleich die Möglichkeit seiner "Erweiterung" hin zum Allgemeinsten überzeugend nachzuweisen. Die Analyse des Geldes wird dabei nicht nur zum Paradigma für eine allgemeine kulturwissenschaftliche Theorie der symbolischen Formen, wie sie später von Ernst Cassirer weiterentwickelt worden ist, sondern bildet zugleich das Programm für eine genuine Weltbildanalyse, welche vermittels einer Rekonstrukion der "Korrelationen" und "Analogien" zwischen den theoretischen und praktischen Erscheinungsformen des modernen Rationalismus und Intellek-tualismus den heuristischen Bezugsrahmen für die späteren kultursoziologischen Arbeiten von Werner Sombart, Max Weber, Karl Mannheim u.a. vorgegeben hat.

Diese Herstellung eines "Gesamtbildes" der einzelnen seelischen Inhalte und der äußeren Erscheinungswelt ist aber eine Aufgabe, die Simmel nicht einer einzelwissenschaftlichen Form der Wirklichkeitserkenntnis abverlangt, sondern als legitimes philosophisches Anliegen ansieht. Denn die durch die moderne Geldwirtschaft geprägte Form der subjektiven Erfahrung von Wirklichkeit ist viel zu sehr in sich fragmentarisch gebrochen, als daß sie die Möglichkeit eines restlosen begrifflichen Erkennens garantieren könnte. Gerade die geldwirtschaftlich bedingte "Distanz" zu den Dingen und die mit ihr einhergehende "Hyperästhesie" impliziere insofern den Reiz des Fragmentes, der bloßen Andeutung, des Aphorismus und des Symbolischen. Simmels 1908 erschienene soziologische Schriftensammlung trägt dieser Eigenart der modernen Wirklichkeitserfahrung deshalb dahingehend Rechnung, daß sie auf eine vorschnelle Systematisierung ihrer Untersuchungsergebnisse bewußt verzichtet und sich in methodischer Hinsicht auf eine Ansammlung von Beispielen, in inhaltlicher Hinsicht dagegen auf eine Zusammen-stellung von Fragmenten beschränkt. Und seine "Philosophie des Geldes" tut dies dergestalt, daß sie auf symbo-lischem Wege, d.h. in Gestalt einer Weltbildanalyse eine Annäherung an jene "Ganzheit des Lebens" versucht, die sich sowohl einer rein empirischen als auch einer begrifflich-rationalen Erfassung entzieht.

Im letzten Kapitel seiner "Philosophie des Geldes" hat Simmel dem durch die Geldwirtschaft geprägten "Stil des Lebens" eine ausführliche Untersuchung gewidmet. Er beschreibt dabei die Kategorie der Distanz als eine räumliche, Rhythmus und Symmetrie als eine zeitlich-räumliche und das Tempo als eine zeitliche Symbolik bzw. "Analogie" des modernen Lebensstils. Im Rahmen seiner Analyse der spezifisch temporalen Erfahrung einer Beschleunigung aller Lebensvollzüge innerhalb der Moderne kommt dabei dem gegensätzlichen Verhältnis zwischen dem Begriff der Beharrung und dem der Veränderung ein ausgezeichneter Stellenwert innerhalb seiner Rekonstruktion des modernen Weltbildes zu. Simmel stellt in diesem Zusammenhang drei unterschiedliche 
Ausprägungen dieses Gegensatzes zwischen der "Form des Beharrens" und der "Form der Bewegung" einander gegenüber, um die prinzipiellen Möglichkeiten einer temporalen Strukturierung von Weltbildern zu verdeutlichen. Wird die Welt nämlich als unveränderliche Substanz aufgefaßt, so muß zumindest eine unaufhörliche Veränderung der Formen angenommen werden, in der sich diese Substanz ausdrückt, soll dem dynamischen Charakter alles Geschehens Rechnung getragen werden können. Werden dagegen die realen Elemente als in fortdauernder Bewegung angesehen, so sind es umgekehrt die Formen selbst, denen ein entsprechendes Beharrungsvermögen zugesprochen wird. Eine dritte mögliche Ausgestaltung dieses Gegensatzes besteht Simmel zufolge aber darin, daß einer "absoluten Form des Beharrens", wie sie durch die neuzeitliche Idee des Naturgesetzes zum Ausdruck gebracht wird, eine entsprechende absolute Form der Bewegung gegenübergestellt wird, welche in der permanenten Zrikulation des Geldes am reinsten zum Ausdruck kommt und sich einem bestimmten Zeitmaß überhaupt entzieht. Simmel sieht in dieser Vorstellung einer solchen "absoluten Veränderung" bzw. einer "species aeternitatis mit umgekehrten Vorzeichen" aber gerade die spezifische temporale Struktur des modernen Weltbildes, in welchem der traditionelle Begriff der Dauer durch eine reine "Form des Übergangs" bzw. der Nicht-Dauer ersetzt worden ist. Indem das Geld als actus purus zum Träger einer Bewegung avanciert, "in dem eben alles, was nicht Bewegung ist, völlig ausgelöscht ist", wird es so zum adäquaten Symbol für diese "allgemeine Relativität der Welt", welche sowohl unser intellektuelles Weltbild als auch unser praktisches, ökonomisches und gefühlsmäßiges Verhalten prägt.

Simmels Anspruch war es, an einem konkreten Gebilde der historischen Welt die allgemeine Signatur des modernen Zeitalters zu veranschaulichen. Daß er im Gelde eine "Weltformel" zu finden hoffte, welche zugleich den "relativistischen Charakter des Seins" in umfassender Weise widerspiegelt, war bei ihm nicht durch ein historisch-genetisches Erkenntnisinteresse, sondern durch das Bedürfnis nach einer einheitlichen philosophischen Interpretation der spezifischen Erfahrungsgehalte der Moderne motiviert. Eine Beantwortung der Frage, ob ihm dieses in einer uns auch heute noch überzeugenden Form gelungen ist, hängt sicherlich stark davon ab, in welchem Ausmaß wir die von ihm aufgestellten Kriterien für eine philosophische Zeitdiagnose nach wie vor zu teilen bereit sind. Daß die Entwicklung von Weltbildern auch in einer historisch-soziologischen Art und Weise untersucht werden kann, haben entsprechende kultur- und wissenssoziologische Forschungen im Anschluß an die Arbeiten von Max Weber und Karl Mannheim gezeigt. Mit Simmel ist jedoch daran festzuhalten, daß einer philosophischen Analyse von Weltbildern nicht nur eine heuristische Funktion für die Entwicklung einer entsprechenden historisch-empirischen Forschungsprogrammatik zukommen kann, sondern einen bleibenden eigenständigen Wert besitzt, wenn sie in der Lage ist, unsere eigenen Bedürfnisse nach einer epochalen Standortvergewisserung unserer gegenwärtigen Zeiterfahrung mit den entsprechenden Antworten der philosophischen Überlieferung zu konfrontieren. In diesem Sinne ist auch heute noch der genuin philosophische Anspruch von Simmels "Philosophie des Geldes" ernst zu nehmen.

In: Simmel Newsletter 3 (1993), S. 99-108. Wiederabgedruckt in: Achim Eschbach / Ernest W. B. Hess-Lüttich (Hrsg.): Das Geld als Zeichen. CODICAS/CODE. Ars Semeiotica 25 (2002), Nr. 3-4, S. 345-354.

(C) 2001-2003 Fachbereich Gesellschaftswissenschaften, Johann Wolfgang Goethe-Universität Frankfurt/Main 